\title{
Adaptive Lighting System as a Smart Urban Object
}

\author{
Michael Aleithe \\ Leipzig University, Germany \\ Email: aleithe@wifa.uni- \\ leipzig.de
}

\author{
Philipp Skowron \\ Leipzig University, Germany \\ Email: skowron@wifa.uni- \\ leipzig.de
}

Bogdan Franczyk

Wroclaw University of Economics, Poland

Email:

bogdan.franczyk@ue.wroc.pl

\author{
Eric Schöne \\ Eric Schöne Chartered Surveyor, \\ Germany \\ Email: es@schoene.co
}

\begin{abstract}
In this article we present an approach to an adaptive lighting system as an intelligent object supporting urban space, especially for the elderly. This intelligent lighting system is used as an instrument to improve the feeling of safety in everyday life by overcoming barriers such as dark areas at night. The intelligence of this system is based on a personalized and position-dependent adaptation of light, whereby intensity and color can be varied. This article focuses on the technical implementation of a corresponding lighting system. In this context, the main point of emphasis is the overall architecture, especially from the point of view of an application system.
\end{abstract}

\section{INTRODUCTION}

$\mathrm{W}$ ith regard to the digitalization of the urban environment, commonly referred to as Smart City, recent efforts in this area are now focusing on an increasingly ageing population. The latest findings will be described in [1] in this context. So-called smart urban objects can be used in public areas to increase the sense of security of older people, which can enhance their participation in public life. In [1] an information radiator is presented as a typical member of a smart urban object whose task is to boost the feeling of well-being by means of a tailored information supply for elderly people.

As another typical representative of a smart urban object, this work realizes a lighting system which aims to increase the feeling of security in this environment by a personalized adaptation of the light. The application portfolio of this adaptive lighting system is aimed at the personalized routing of elderly people, especially in rural areas, since the light pollution component is initially negligible due to too many people on one lighting section. The focus of this work is on the conception of a complete technical architecture as well as the description of the individual sub-components, which are necessary in the course of an implementation. So we can formulate the following research question: How must an application architecture be designed in order to implement personalized and position-dependent adaptive lighting?
The aspect of personalization is realized by a personal transmitter, which can be comfortably carried along by the elderly and can be used for distance measurement. Based on this personalized distance information, the light can be adapted to the intensity and color of the light. The psychological component of this light adaptation is excluded from the investigations in this article.

Section 2 of this article explains the preliminary work to date in the field of intelligent lighting systems. Subsequently, Section 3 describes the general technical system design with all associated subsystems for the implementation of a personalized adaptive lighting system. Based on this, a detailed examination of the specific elements of the subsystems required for an implementation is carried out in Section 4. Section 5 explains the advantages and disadvantages of the system, which can be evaluated based on the implemented prototype. In summary, Section 6 concludes with a final review of the adaptive lighting system.

\section{Related Work}

In the past, various attempts tried to adjust the effect of light on adequate situations of everyday life in a target-oriented way. In [2], lighting in buildings was optimized so that sensors first register the existing natural light and, based on the acquired information, afterwards reduce the energy-bound electrical lighting to a minimum.

The minimized energy consumption also reduced the energy costs. This approach used wireless sensor networks in order to detect the already existing daylight. In contrast to the wire-bound variant, the wireless option showed various advantages. On the one hand, higher flexibility is given which makes the installation of the option much easier in already existing buildings. Further, the size and costs of the metering sensor system are being reduced due to the wireless variant. The network consists of an array of light sensors which communicate with a master node. That way, information about the current lighting state of the daylight are provided. The controlling of the lamps happens based on the master node. 
A polychromatic lighting system, which is variable in terms of light color and intensity of lighting, was introduced by [3]. This way, lighting can be adjusted adequately to the needs of the user. Based on the already existing lighting in tensity and light color, the realization of the light constellation is being regulated via linear and non-linear optimization techniques so that only a minimum of energy is needed. In order to apply optimization strategies in this case, the overall system had to be modelled. This includes measuring elements and lighting elements. The lighting elements include the already existing daylight, light bulbs, fluorescent lamps as well as LED arrays, though only the latter can be regulated in terms of lighting intensity and light color. The metering is done by sensor nodes and includes the recording of the light temperature of all light elements and the transmission of the information to the Controller. Optimization calculations are being done on the Controller in order to create a base for the regulation of the LED arrays and consequently the desired lighting constellation.

Based on the trend of using the already existing daylight more efficiently and thus to economize more ecologically and sustainably, [4] justify the so called connected lighting as an essential component for Smart Cities. The transfer from a traditional to an intelligent lighting was described. It was pointed out that the intelligence of this transfer is due to the interlinking of the individual lighting elements and thus the internal communication. The substantial advantages of the intelligent lighting in contrast to the traditional variant are remote monitoring, the technical components, a smart asset management as well as a constantly optimized reduction of lighting energy in order to economize more energy-efficiently. The result is a reduction of the lighting intensity of lamps on much frequented roads during quiet phases. In this source, the lighting concept which is connected to the internet is referred to as "Energy-Internet."

Smart lighting was used by [5] as a component to support sustainability and to improve quality of life of the participating populations in the Smart City context. Thus, a lighting system, which controls the street lights energy-efficiently without affecting the traffic situation or the social components of the area in a negative way, was presented. Smart lighting aims at operating adaptively and autonomously. The system includes intelligent controlling of street lights, which is based on adaptive behavioral rules. The traffic situation of the concerned area was registered via wireless sensor networks. This strategy was evaluated through an agent-based simulation. The result was a reduction of the spent lighting energy by 33\%.

[6] investigated the realization of the light source adaption. Focus of the investigation, in this case, was on the minimization of the energy consumption. The lighting intensity was adapted via a regulation - nominal value and real value of the lighting intensity are being compared continuously and in case of a discrepancy, the real value is being adjusted. The logic of rules is derived from fuzzy techniques.

[7] conducted a study in which various strategies for adaptive lighting were put into practice, the resulting energy gain was then analyzed. The portfolio of these strate- gies includes, among other things, aspects of adaptive lighting. In order to realize an adaption to the environment, a sensor-based variation of the lighting intensity takes place. Further, an optimization concerning peak phase and low phase of the traffic to be handled takes place. During the peak phase, the lighting intensity increases and decreases during the low phase. Moreover, the area, which is analyzed in this study, has to be divided into zones and further inspected separately. The results of this study showed a significant reduction of energy costs, partly due to the strategies already introduced.

Another concept, introduced by [8], addresses the energy consumption of exterior lighting in cities. The concept includes a controlling system which, besides a minimized energy consumption, requests a remote diagnosis. That way, additional maintenance costs are expected to be avoided. The applied strategies are orientated towards the effects of the building automation on the building efficiency, as described in EN 15232 in more detail.

Ultimately, an immense saving of energy was proved. Concerning the minimization of the energy consumption of the used lighting components, [9] presented a method which optimizes the lighting intensity through an iterative method. In this context, a specifically centralized lighting control system was designed for this purpose. [10] reached a minimization of the illumination level through the maintenance of specifically defined nominal value and a steady minimization of the electrical energy. A cognitive approach through an adequate regulation algorithm was taken, whereby a non-linear characteristic of the illumination level was taken into consideration. Later, this approach was evaluated experimentally through [11]. In the end, an immense importance for a possible practical use could be proved.

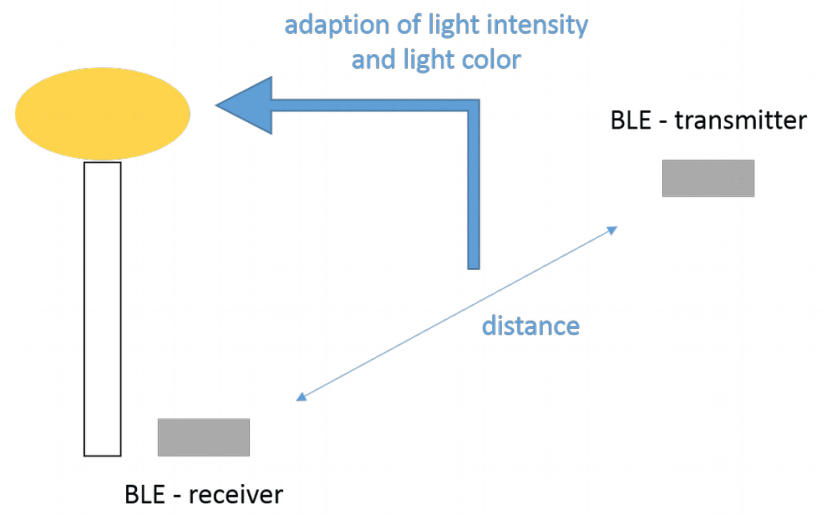

Fig 1. This picture illustrates the schematic overview of the system design in general. As consequence of the distance variability between receiver and transmitter and the coherent variation of BLE signal strength the parameter of the light behavior will be adapted. In this work we focused on intensity and color as light parameters.

[12] introduces a completely remote-controlled street light island as application in the Smart City context. The architecture with its diverse hierarchical structures is being described and the technologies used are being discussed in 
detail. This includes mainly the controlling via Raspberry$\mathrm{Pi}$ and wireless transmission via ZigBee.

In contrast to previous approaches, mainly explaining technical and to some extend economical concerns, [13] and [14] analyze the psychological effect of the lighting system on elderly people. Especially [13] aim at improving the mood of elderly people via adaptive lighting systems. Requirements for a technical system were defined, which detects the mood of elderly people and thereupon adapts the lighting in the room, aiming at activating the person by designing a specific mood-model.

\section{Aproach for System Design}

Based on the research of previous work on intelligent lighting systems (Section 2), it becomes apparent that there is no system for personalized position measurement with coupled adaptation of lighting parameters in public spaces. The system architecture described in this section addresses this particular challenge. In principle, personalized distance measurement is performed using the Bluetooth Low Energy (BLE) standard [15]. A BLE transmitter is assigned to each person. The distance measurement is carried out by assigning a distance-dependent signal strength to the BLE receiver. This signal is called Received Signal Strength Indicator (RSSI). Starting from the incoming RSSI at the BLE receiver, the intensity and/or color of the light is adapted. This general system structure is illustrated in Fig. 1 for better understanding.

\section{IMPLEMENTATION}
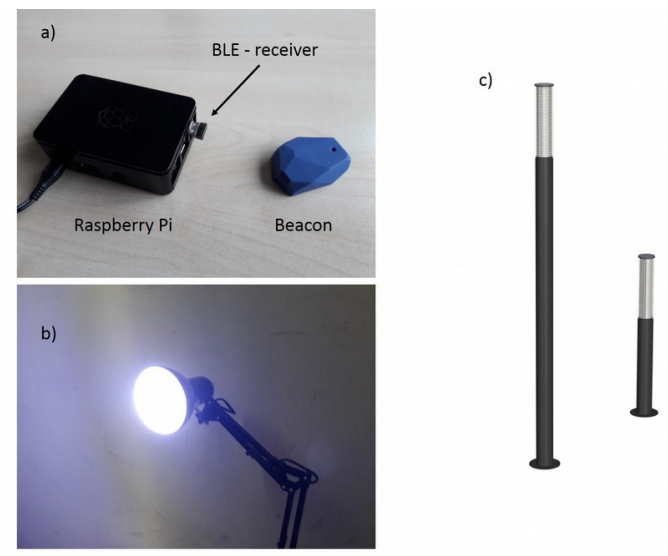

Fig 2. This illustration demonstrates the individual hardware components of the adaptive lighting system. In a) the BLE transmitter and receiver are represented, in b) the prototypical lamp fitting and in c) the lamp body for public space.

An Estimote Beacon is used as a personalized BLE transmitter [16]. The receiver is realized by a Bluetooth 4.0 adapter [17], which is able to receive the BLE standard. The transmission protocol is the so-called Eddystone protocol in UID format [18]. These received data (distance measurement) can then be further processed in a control unit and subsequently the adaptation of the lamps can be realized. Specifically, the lamp is controlled by means of a ZigBee gateway, which can be addressed from the control unit via REST. The Philipps Hue lamps [19] adapt their lighting parameters to the received control data of the ZigBee protocol. The BLE receiver, the control unit and the ZigBee gateway for lamp control are implemented on a Raspberry Pi. There is also a MQTT client on top of it. This serves as a further component for integration into a network and for monitoring purposes. The overall architecture with its individual modules described above is shown schematically in Fig. 3.

The implementation from the hardware perspective is illustrated in Fig. 2. a) contains the comfortable Estimote Beacon, which is used as a personalized BLE transmitter, and the Raspberry Pi with all the subsystems described above. Furthermore, b) shows the prototypical outfit of Philipps Hue lamps as used for test purposes. In public space, these lamps and the Raspberry Pi are integrated into a lamp body as shown in c) [20].

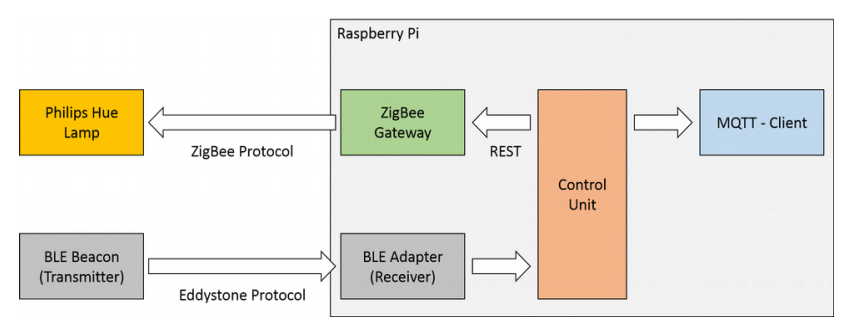

Fig 3. This picture demonstrates the architectural model of the adaptive lighting system with submodules and their communication dependencies.

\section{Results and Discussion}

The system design described in Section 3, which has been discussed in more detail in Section 4 with regard to its technical implementation, is here subjected to an analysis of its advantages and disadvantages.

The implemented prototype is easy to use in several ways. On the one hand, the user does not need to operate the beacon (BLE transmitter) any further. On the other hand, the Beacon can easily be carried along in everyday life. In addition to this ease of use, Beacons generally have a long battery life, which further enhances the usability. The hardware required for the control (Raspberry Pi with subsystems) can be easily integrated into the lamp carcass, which is intended for later use in public spaces as illustrated in Fig. 2 c). Furthermore, a certain degree of flexibility can be observed from a software point of view, since standardized protocols and formats are used throughout (Section 4), so that individual subsystems are relatively easy to replace.

Furthermore, the quality of the BLE signal was examined in detail. In this context, the RSSI was measured depending on the distance between the transmitter and receiver. The measurements were carried out both with and without a lamp body to determine the influence of the additional object on the signal absorption. For each constellation 10 measuring points were recorded. The results are shown in a Box-Plot diagram in Fig. 4. Here, a relatively high variabil- 
ity of the measured values can be observed, which means that an exact distance measurement is not necessary. The causes of these fluctuations can have many different causes, since BLE reacts sensitively to liquids, WLAN and metallic bodies [21, 22, 23], but these cannot be prevented in public space. In general, a signal attenuation through the lamp body can be determined by the measured values. In both cases (with and without body), however, it is possible to differentiate according to the measured values of the RSSI whether a beacon is located inside or outside a radius of about 5 meters around the receiver.

\section{Conclusion and Outlook}

In this work, a novel smart urban object is presented, which is realized by a personalized adaptive lighting system. The focus is on the technological architecture. A concept for an overall architecture and all subsystems involved was presented, which were necessary for the implementation. The completed prototype was briefly presented and evaluated according to various criteria. In summary, it is a personalized adaptive lighting system that provides a distance-dependent variation of the intensity and/or color of the light, whereby the accuracy of the distance measurements is limited to a differentiation between greater or less than 5 meters.

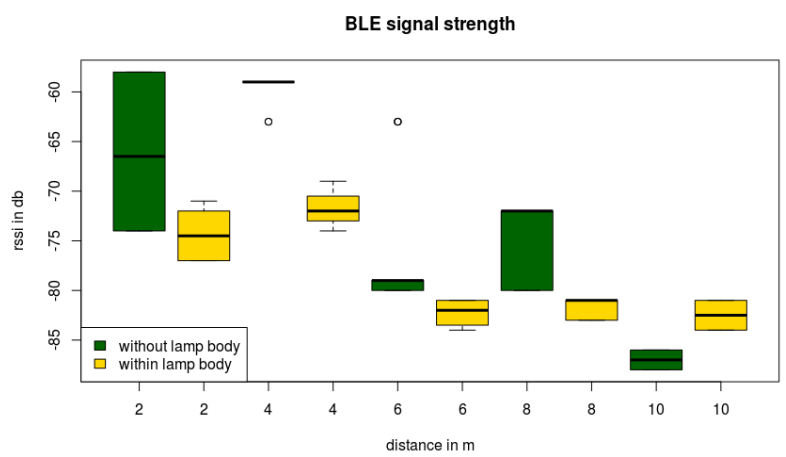

Fig 4. In this boxplot-diagram we illustrate the numeric relation between the Received Signal Strength Indicator (RSSI) of BLE signal and the distance of the BLE -transmitter and -emitter. The measurements conducted with the BLE-sensor without (green) and within (yellow) the lamp body to investigate the influence of the lamp body.

Due to this measurement disturbance, data-technical corrections which increase the precision of measurement will be offered in the future. Also, this lighting system will be installed in a test site that ideally reflects the public space. For this reason, an investigation of the psychological aspects is necessary to what extent adaptive light influences the mood of the participating persons. Furthermore, the realization of a complex network of adaptive lighting stations is aimed at, so that entire areas of the public space can be covered. In addition, the existing lighting system must be expanded to include several people or groups of people.

\section{ACKNOWLEDGMENT}

This work was fully conducted in the scope of the research project UrbanLife + (16SV7442), funded by the German Federal Ministry of Education and Research.

\section{REFERENCES}

[1] Koch, M., Kötteritzsch, A., Fietkau, J.: Information radiators: using large screens and small devices to support awareness in urban space. Proceedings of the International Conference on Web Intelligence (WI '17), pp. 1080-1084. ACM, Leipzig (2017)

[2] Nippun Kumaar, A. A., Kiran, G., Sudarshan, T. S. B.: Intelligent Lighting System Using Wireless Sensor Networks. International Journal of Ad hoc, Sensor \& Ubiquitous Computing (IJASUC) 1 (4), pp. 17-27 (2010). doi:10.5121/ijasuc.2010.1402

[3] Paradiso, J. A., Aldrich, M., Zhao, N.: Energy-efficient control of solidstate lighting. SPIE Newsroom. (2011). doi:10.1117/2.1201102.003543

[4] Crowther, J., Herzig, C., Feller, G.: The Time Is Right for Connected Public Lighting Within Smart Cities. Cisco Internet Business Solutions Group (IBSG) (2012)

[5] Escolar, S., Carretero, J., Marinescu, M., Chessa, S.: Estimating Energy Savings in Smart Street Lighting by Using an Adaptive Control System. International Journal of Distributed Sensor Networks, pp. 1-17. (2014). doi:10.1155/2014/971587

[6] Papantoniou, S., Kolokotsa, D., Kalaitzakis, K., Cesarini, D. N., Cubi, E., Cristalli, C.: Adaptive lighting controllers using smart sensors. International Journal of Sustainable Energy 35 (6), pp. 537-553 (2014). doi:10.1080/14786451.2014.923887

[7] Jackson, H., Jackson, S., Jackson, C., Siminovitch, M.: Saving Energy in Buildings with Adaptive Lighting Solutions. California Energy Commission. California Lighting Technology Center, UC Davis (2015)

[8] Ożadowicz, A., Grela, J.: Energy saving in the street lighting control system - a new approach based on the EN-15232 standard. Energy Efficiency (2016). doi:10.1007/s12053-016-9476-1

[9] Caicedo, D., Pandharipande, A.: Daylight and occupancy adaptive lighting control system: An iterative optimization approach. Lighting Research and Technology 48 (6), pp. 661-675 (2016), doi:10.1177/1477153515587148

[10] Yin, C., Stark, B., Chen, Y., Zhong, S.: Adaptive minimum energy cognitive lighting control: Integer order vs fractional order strategies in sliding mode based extremum seeking. Mechatronics 23 (7), pp. 863872 (2013). doi:10.1016/j.mechatronics.2013.09.004

[11] Yin, C., Stark, B., Chen, Y., Zhong, S., Lau, E.: Fractional-order adaptive minimum energy cognitive lighting control strategy for the hybrid lighting system. Energy and Buildings 87, pp. 176-184 (2015). doi:10.1016/j.enbuild.2014.11.036

[12] Leccese, F., Cagnetti, M., Trinca, D.: A smart city application: a fully controlled street lighting isle based on Raspberry-Pi card, a ZigBee sensor network and WiMAX. Sensors 14 (12), pp. 24408-24424 (2014). doi:10.3390/s141224408

[13] Huldtgren, A., Katsimerou, C., Kuijsters, A., Redi, J. A., Heynderickx, I. E. J.: Design Considerations for Adaptive Lighting to Improve Seniors' Mood. Inclusive Smart Cities and e-Health, Bd. 9102. Cham: Springer International Publishing (Lecture Notes in Computer Science), pp. 1526 (2015)

[14] Kuijsters, A., Redi, J., Ruyter, B. d., Heynderickx, I.: Lighting to Make You Feel Better: Improving the Mood of Elderly People with Affective Ambiences. PloS one 10 (7) (2015). doi:10.1371/journal.pone.0132732

[15] Specifications: The building blocks of all Bluetooth devices. https://www.bluetooth.com/specifications. Accessed on 3 Jan 2018

[16] The Physical World: Software-defined. https://estimote.com/. Accessed on 3 Jan 2018

[17] Bluetooth $4.0 \quad$ USB Adapter. https://www.asus.com/Networking/USBBT400/. Accessed on 3 Jan 2018

[18] Eddystone-UID.

https://github.com/google/eddystone/tree/master/eddystone-uid. Accessed on 3 Jan 2018

[19] White and colour ambience. https://www.philips.co.uk/cp/8718696461679/hue-white-and-colour-ambience. Accessed on 3 Jan 2018

[20] https://www.burri.shop/resources/Public_Light_small_reflektor.jpg. Accessed on 3 Jan 2018

[21] Estimote Beacons: Will wireless interference and Wi-Fi impact beacons? https://community.estimote.com/hc/en-us/articles/200794267-Willwireless-interference-and-Wi-Fi-impact-beacons-. Accessed on 3 Jan 2018 
[22] Estimote Beacons: Best practices for installing Estimote Beacons. https://community.estimote.com/hc/en-us/articles/202041266-Bestpractices-for-installing-Estimote-Beacons. Accessed on 3 Jan 2018
[23] Estimote Beacons: What are Broadcasting Power, RSSI and other characteristics of a beacon's signal? https://community.estimote.com/ hc/en-us/articles/201636913-What-are-Broadcasting-Power-RSSIand-other-characteristics-of-a-beacon-s-signal-. Accessed on 3 Jan 2018 